\title{
Targeting Poison Exons to Treat Developmental and Epileptic Encephalopathy
}

\author{
Miriam C. Aziz ${ }^{a}$ Patricia N. Schneidera,b Gemma L. Carvilla, c \\ aKen and Ruth Davee Department of Neurology, Northwestern University School of Medicine, Chicago, IL, USA; \\ ${ }^{b}$ Instituto de Ciencias Biologicas, Universidade Federal do Para, Belem, Brazil; ' Department of Pharmacology and \\ Pediatrics, Northwestern University School of Medicine, Chicago, IL, USA
}

\section{Keywords}

Epilepsy · Alternative splicing · Poison exons · Antisense oligonucleotide $\cdot$ Genetics

\begin{abstract}
Developmental and epileptic encephalopathies (DEEs) describe a subset of neurodevelopmental disorders categorized by refractory epilepsy that is often associated with intellectual disability and autism spectrum disorder. The majority of DEEs are now known to have a genetic basis with de novo coding variants accounting for the majority of cases. More recently, a small number of individuals have been identified with intronic SCN1A variants that result in alternative splicing events that lead to ectopic inclusion of poison exons (PEs). PEs are short highly conserved exons that contain a premature truncation codon, and when spliced into the transcript, lead to premature truncation and subsequent degradation by nonsense-mediated decay. The reason for the inclusion/exclusion of these PEs is not entirely clear, but research suggests an autoregulatory role in gene expression and protein abundance. This is seen in proteins such as RNA-binding proteins and serine/arginine-rich proteins. Recent studies have focused on targeting these PEs as a method for therapeutic intervention. Targeting PEs using antisense oligonucleotides (ASOs) has shown to be effective in modulating alternative
\end{abstract}

splicing events by decreasing the amount of transcripts harboring PEs, thus increasing the abundance of full-length transcripts and thereby the amount of protein in haploinsufficient genes implicated in DEE. In the age of personalized medicine, cellular and animal models of the genetic epilepsies have become essential in developing and testing novel precision therapeutics, including PE-targeting ASOs in a subset of DEEs.

(c) 2021 S. Karger AG, Basel

\section{Introduction}

Alternative splicing of the transcriptome underlies much of the protein complexity in the human brain, enabling the generation of a diverse set of proteins from essentially the same set of coding regions in all higher order mammals. In addition, alternative splicing of noncoding exons can impact the amount of functional protein within the cell. These noncoding exons are known as poison exons (PEs); they are typically small (<100 bp), highly conserved exons that contain an in-frame stop codon. When incorporated into a particular transcript, the introduction of this premature truncation codon triggers nonsense-mediated decay (NMD) and degradation of the mRNA [1]. For this reason, PEs are often referred to as NMD exons. Inclusion of PEs in a transcript results in karger@karger.com www.karger.com/dne

(C) 2021 S. Karger AG, Base

Karger"
Correspondence to:

Patricia N. Schneider, patricia.schneider@ nort.wes.ern.edu Gemma L. Carvill, gemma.carvill@ northwestern.edu 
Fig. 1. PE exclusion and inclusion are associated with productive and nonproductive splicing, respectively. $\mathrm{PE}$, poison exon; PTC, premature truncation codon; NMD, nonsense-mediated decay.

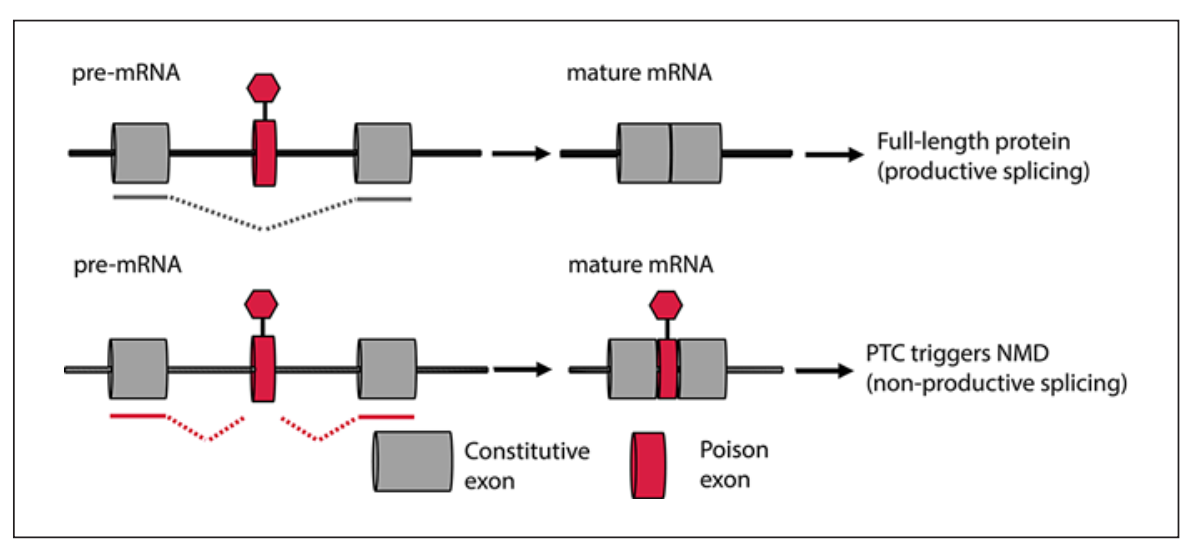

unproductive splicing, whereas skipping/exclusion of PEs results in productive splicing (Fig. 1).

The function of these PEs is not entirely clear, but PEs likely control the amount of functional protein within a cell in a much more rapid manner than can be achieved by activating and silencing transcription. For instance, RNA-binding proteins (RBPs), especially serine/arginine-rich (SR) proteins autoregulate their own transcription levels through the inclusion of a $\mathrm{PE}$ that triggers NMD. This autoregulatory mechanism negatively regulates SR transcription, thus maintaining homeostasis of SR proteins and SR-regulated proteins in the cell [2].

PEs tend to occur in ultra-conserved elements (UCEs) in the genome. The human genome harbors several hundred UCEs, some of which have been shown to function as transcription enhancers and regulate gene expression [3], but many are also found in introns and can function as PEs. For instance, phylogenetic analysis of ancient SR proteins has shown that SRSF5 contains an UCE that is conserved across vertebrates and presents sequence similarity to drosophila and fungi. Moreover, PEs in SR proteins have been retained even after subsequent duplication events involving SR genes in vertebrates, suggesting an important role in regulation of gene expression. Despite differences in NMD mechanisms among species, the cellular role of alternative unproductive splicing of SR proteins is believed to be coupled with the NMD pathway [2, 4].

PEs are also important in neuronal development, and alternative splicing of these exons is tightly regulated by coordinated exon usage. In mice, RBPs, such as Ptbp 1 and Rbfox, act antagonistically to regulate the inclusion/exclusion of a PE in Flna, thus maintaining the neural progenitor and neuron identity. Disruption of the PTPB1binding site in FLNA in humans leads to brain malformations. PEs have been extensively studied in the context of RBPs, and, in particular, SR proteins, and the presence of pathogenic variants within and around PEs in SR genes and also in functionally unrelated genes has been linked to neurodevelopmental disorders (NDDs) [5]. Finally, the misregulation of PE splicing has also been identified in individuals with Duchenne muscular dystrophy, Ewing sarcoma, and other types of cancer [6-9].

While there are indeed many genes important in neurodevelopment and function that harbor PEs, this review centers around the PEs that occur in genes associated with epilepsy and specifically the developmental and epileptic encephalopathies (DEEs). The DEEs are a heterogeneous group of rare NDDs, generally characterized by early-onset seizures that are often intractable, and cognitive features such as developmental delay, plateau, or regression. We discuss genetic variants that perturb alternative splicing of PEs, which DEE-associated genes harbor these exons, and the role of PEs as putative therapeutic targets in the DEEs.

\section{Genes Implicated in Epilepsy That Harbor PEs}

Nearly 20 years ago, a PE called $18 \mathrm{~N}$ (N-neonatal) was identified in the voltage-gated sodium channel gene, Scn8a. This PE is included in non-neuronal cells and throughout early fetal brain development but is excluded later in neuronal development and throughout life, where full-length Nav1.6 (SCN8A) is required for proper neuronal function $[10,11]$. This PE is highly conserved between mice and humans and can be detected in human nonneuronal cells (Fig. 2). Only later, gain-of-function (GOF) missense de novo variants were identified in SCN8A in association with a severe DEE $[12,13]$. To date, no pathogenic variants have been identified that disrupt splicing of the $18 \mathrm{~N}$ SCN8A PE. Conversely, pathogenic variants that disrupt PE splicing have been identified in individuals with another DEE, Dravet syndrome, typically caused by 
de novo loss-of-function (LOF) variants in SCN1A. In this study, 5 individuals with Dravet syndrome carried likely pathogenic variants in, or near, the SCN1A PE, 20N, and the presence of a subset of these variants resulted in increased inclusion of $20 \mathrm{~N}$ ( $\mathrm{N}$-nonsense) in a minigene assay. We proposed that this increased PE inclusion leads to NMD in the neurons of affected individuals and essentially SCN1A haploinsufficiency [14]. In this instance, the identification of the PE was driven by the presence of genetic variants in a highly conserved intronic region but sparked renewed interest in the identification of similar PEs in epilepsy genes using transcriptomics.

Accordingly, transcriptomic analysis of fetal brain tissue $[11,15]$, as well as our own work in induced pluripotent stem cell (iPSC)-derived neurons and non-neuronal cell lines (unpublished), has revealed candidate PEs in epilepsy-associated genes. The genes most commonly implicated in epilepsy are SCN1A, KCNQ2, MECP2, SCN2A, STXBP1, PCDH19, DEPDC5, SYNGAP1, CHD2, and SCN8A [16-18]. Of these "top 10" genes, the majority harbor potential PEs, including all of the sodium channels, as well as SYNGAP1 and CHD2 (Fig. 2). These PEs include cassette exons, as well as alternative $5^{\prime}$ and $3^{\prime}$ splice sites that incorporate a premature truncation codon (Fig. 2). However, only SCN1A, SCN8A, and $S Y N G A P 1$ PEs have been validated by other methods, and only for SCN8A, the RBPs that control alternative splicing have been established $[10,14]$. Future studies will be important to determine which of these PEs are indeed "real" versus transcriptional noise, as well as what the function of PEs during neuronal development and function are.

\section{Targeting PEs with Antisense Oligonucleotides as Treatment}

These PEs are important potential targets for antisense oligonucleotides (ASOs) (described below), and preventing $\mathrm{PE}$ inclusion and thus increasing the amount of full-length transcript is a treatment for genetic epilepsies with a haploinsufficient disease mechanism (Fig. 3a). Potential target genes include SCN1A, SYNGAP1, and CHD2 that harbor PEs and are likely to cause DEE by haploinsufficiency.

ASOs are short fragments of DNA that naturally bind to specific mRNAs via canonical base-pairing. Throughout their development, ASOs have been chemically modified to increase resistance to nucleases, prevent immune response, and increase binding affinity to the target [19]. The most recent generation of ASOs used to modulate splicing is generally $12-30$ nucleotides in length in the

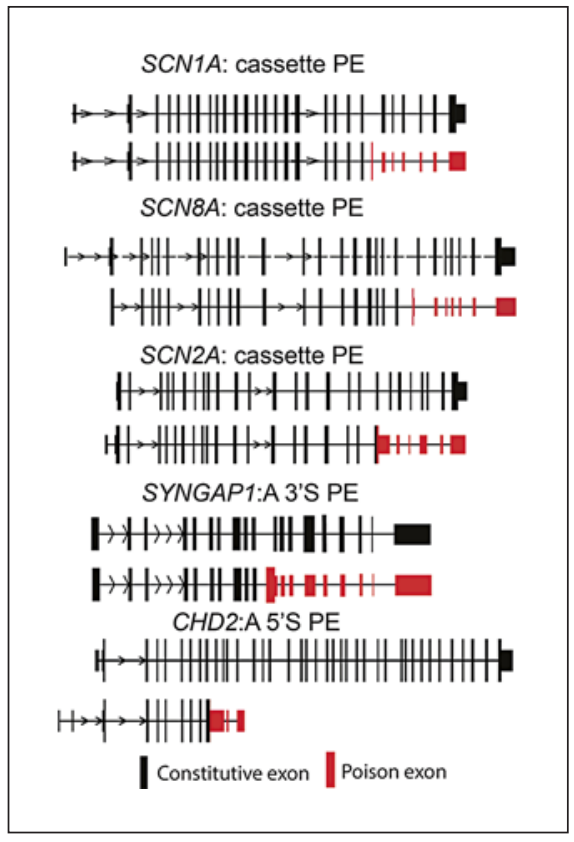

Fig. 2. Epilepsy-associated genes that harbor candidate and known PEs. For each gene, the full-length target (black - top) is shown in comparison to the transcript which harbors the PE; constitutive exons (black) are largely overlapping, until the splicing of the PE (red) which is followed by a multiexon $3^{\prime} \mathrm{UTR}$. PE, poison exon.

form of a gapmer, a hybrid of a phosphorothioate backbone and $2^{\prime}$-O-methoxyethyl ribose substitution, both of which contribute to the ability of the ASO to modulate splicing. These modifications to increase stability and binding are critical to the application of targeting PEs to increase/decrease productive protein [19-21].

ASOs can be targeted to induce alternative splicing of PEs; for instance, targeted augmentation of nuclear genetic output (TANGO) was recently developed by Stoke Therapeutics. Because there is always a small amount of premRNA that generates nonproductive mRNA, TANGO can be used to increase the amount of protein by blocking PE inclusion. Importantly, the ASO cannot discriminate between the transcripts that carry the reference sequence and the pathogenic variant, and so blocking PE inclusion essentially leads to increased production of full-length transcripts of both alleles, even though one may be nonfunctional (i.e., the transcript with the pathogenic variant) (Fig. 3a). Despite this caveat, we give an example below where the amount of protein can still be increased in haploinsufficient disorders.

Using transcriptomic analysis, 2 epilepsy-associated genes, SCN1A (20N, Fig. 2) and SYNGAP1, were found to harbor PEs and thus are good targets for the TANGO technology. These PEs were validated using cycloheximide to 


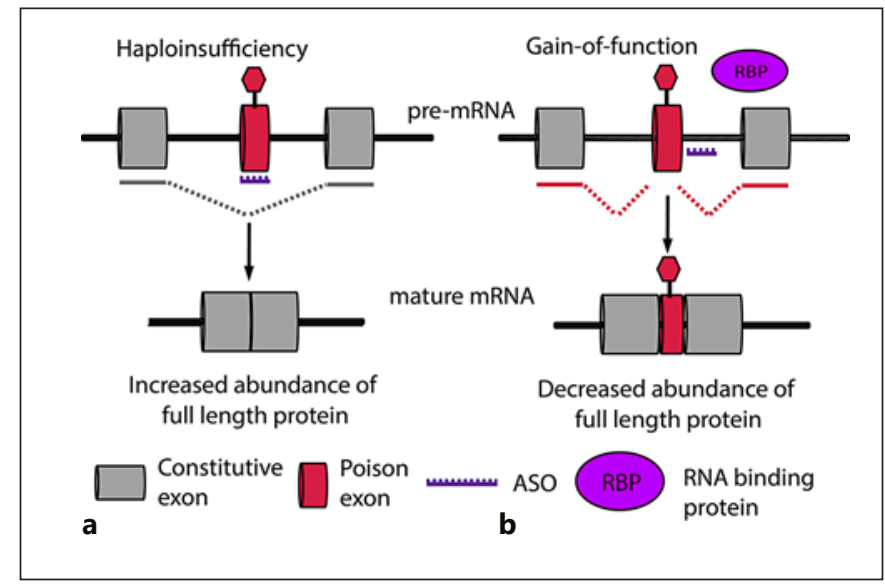

Fig. 3. Potential ASO therapeutic approaches targeting PEs in the DEEs. a Treatment of haploinsufficient disorders, restoring wildtype protein levels: an ASO is designed with complementary base pairs to the PE, preventing its inclusion in the transcript and thus promoting productive splicing of the full-length transcript. b Treatment of GOF disorders, reducing protein levels: blocking the binding of RBPs that prevent the inclusion of a PE is a theoretical approach to promoting PE inclusion and NMD of the transcript and thus reducing protein levels. ASO, antisense oligonucleotides; PE, poison exon; GOF, gain-of-function; RBPs, RNA-binding proteins; NMD, nonsense-mediated decay; DEE, developmental and epileptic encephalopathies.

inhibit translation followed by quantitative RT-PCR, which showed an increased presence of the PE when NMD is inhibited. To identify putative effective ASOs, tiling of ASOs across the PE and flanking regions was performed. Using this approach, ASOs were identified that reliably reduce PE inclusion in a cellular model (ReN cells) and increase both mRNA and protein levels. Moreover, because SCN1A exon 20N in humans is homologous to mouse exon $21 \mathrm{~N}$ [14], ASO target engagement was validated in vivo. P2 wild-type C57BL/6 N mice received a single intracerebroventricular (ICV) injection of the ASO. Analysis of coronal brain slices 5 days post-ICV injection indicated increases in productive Scnla mRNA transcripts with decreases in nonproductive transcripts as well as an increase in $\mathrm{Na}_{\mathrm{v}} 1.1$ abundance, validating the use of an SCN1A-PE targeting ASO in vivo to modulate protein expression [19].

Currently, individuals with Dravet syndrome endure refractory seizures, owing to the inefficiency of anti-seizure medications [22]. The TANGO ASO technology provides a unique approach to modulate splicing in SCN1A haploinsufficiency and was tested in a mouse model developed by the Kearney lab, Scn1 $a^{\text {tmlKea }}$, in which exon 1 was deleted. This mouse model effectively recapitulates the human Dravet syndrome phenotype as it exhibits spontaneous seizures and is highly susceptible to sudden unexpected death in epilepsy [23]. Scn 1a ${ }^{\text {tm1Kea }} \mathrm{P} 2$ mice were treated with ASO-22 via a single ICV injection. Notably, a 90-day survival analysis demonstrated $97 \%$ survival of ASO-treated Scn1 $a^{\text {tmlKea }}$ mice. At 7 and 14 weeks, ASO-22 was still present at concentrations sufficient to increase Scn1 1 transcript levels and $\mathrm{Na}_{\mathrm{v}} 1.1$ protein abundance to wild-type levels. A separate cohort of $S c n 1 a^{\text {tm } 1 K e a}$ mice received a single $20-\mu \mathrm{g}$ ICV injection of ASO-22 at P2. At P20, mice underwent surgery to install EEG headmounts which monitored seizures from P22 to P46. EEG data indicated ASO-22-treated Scn $1 a^{\text {tm } 1 \text { Kea }}$ mice experienced fewer seizures, and latency to first seizure was prolonged [24].

Together, these studies provide preclinical evidence that ICV administration of TANGO ASO-22 increases productive Scn1a mRNA transcripts and $\mathrm{Na}_{\mathrm{v}} 1.1$ protein in Scn $1 a^{+/-}$mouse brains to wild-type levels. ASO-22 works by preventing the exclusion of exon $20 \mathrm{~N}$ ( $21 \mathrm{~N}$ in mice) that normally leads to NMD and has been shown to safely and effectively increase survival in a mouse model of Scn 1 a haploinsufficiency. Moreover, the first patients were recently enrolled in the MONARCH clinical trial to investigate the efficacy, safety, and pharmacodynamics of STK-001 (ASO22) administration in humans (NCT04442295).

In addition to blocking PE inclusion, promoting the inclusion of PEs may also be a potential ASO therapeutic target. Promoting exon inclusion using an ASO is not as straightforward as blocking inclusion but is possible with a good understanding of the RBPs that control splicing. For instance, by blocking the binding of an RBP that prevents inclusion of an exon, exon inclusion is promoted (Fig. 3b). This approach has been successfully implemented in spinal muscular atrophy with the ASO called nusinersen [25]. Here, nusinersen targets the RBP-binding site near exon 7 of the SMN1 gene, inhibiting the binding of hnRNP and thus promoting the inclusion of this exon. Subsequently, the inclusion of this exon results in a full-length transcript. This approach requires a more concise understanding of the RBPs that promote exclusion and inclusion of these PEs and may be guided by the identification of patient-specific variants that disrupt this process as in the example of $S C N 1 A$ [14]. Genes such as SCN8A that harbor PEs and GOF in the pathogenic mechanism are good candidates for this approach particularly as some of the RBPs that control inclusion/exclusion of this exon (18N) are already known [11].

In this review, we and indeed the epilepsy field in general, have focused primarily on the channelopathies, as highlighted in the SCN1A/SCN8A examples previously mentioned. In these instances, restoration of protein levels 
to physiological levels can rescue the disease-associated phenotypes in preclinical models. However, whether this paradigm will be efficient for other DEE-associated genes that play important roles in neurodevelopment (e.g., CHD2 and SYNGAP1) remains to be determined. This is true not only for PE-targeting ASOs but also for any precision therapies for these disorders. Here, animal and stem models will be important for determining the developmental window for interventions. Finally, for many of the genetic epilepsies, affected individuals can indeed have a period of normal development, and regression or plateau of cognitive skills occurs with seizure onset. This suggests that assuming a genetic diagnosis is made early, precision therapies may be effective in preserving the developmental trajectories of individuals with these genetic conditions.

\section{Models for Testing Efficacy of Future ASOs: Mouse Models and iPSCs}

Many of the initial proof-of-principle experiments to test the ability of an ASO to restore physiological protein levels can be performed in cellular models, while the efficacy of ASOs to rescue phenotypes (e.g., seizures) are, at present, constrained to animal models. These preclinical studies have been performed in SCN1A haploinsufficient cell and rodent models, and for other DEEs, there are indeed rodent models that recapitulate the phenotypes seen in humans, for instance, the $\operatorname{Scn} 8 a^{R 1872 W /+}$ mouse, which recapitulates early seizure onset and susceptibility to sudden unexpected death in epilepsy via a GOF mechanism, as well as the Syngap $1^{+/-}$mouse, which recapitulates early-life recurrent seizures that proceed into adulthood via a LOF mechanism [26, 27]. For others, including CHD2, rodent models do not have seizures, though they do have learning and memory deficits [28].

Induced pluripotent stem cells (iPSCs) have become an important tool to study cell-type-specific molecular mechanisms involved in NDDs including epilepsy [29]. The ability to differentiate iPSCs into various neuronal models, including neural progenitor cells, neurons, and astrocytes, allows us to study a variety of time points during development, as well as to define the developmental window for targeting PEs [30]. These versatile cells can also be used to generate a 3D cellular model by differentiating into cerebral organoids, which recapitulate many of the cellular populations and organizational patterns seen in human brains [31].

Patient-specific lines allow us to functionally assay suspected pathogenic mechanisms following a genetic diagno-

Poison Exons in Developmental and

Epileptic Encephalopathy sis, and developing isogenic controls from patient lines allows us to study disease-associated variants in the same genetic background. Using patient-specific iPSC models is useful for drug development and testing as it enables us to study the effects of therapeutics on various populations of cells that are specific to the patient's genotype and phenotype. Moreover, some studies have shown that iPSC models can display neurophysiological abnormalities using multielectrode arrays and patch-clamp electrophysiology. For instance, in a model of $S C N 8 A$-associated DEE, the use of voltage-clamp electrophysiology and multielectrode arrays has been shown to reliably detect persistent sodium current in patient iPSC-derived neurons and can detect rescue of the current to control levels upon treatment with antiseizure medications [32]. This technology, while still in its infancy, is another platform for future testing of ASOs.

\section{Conclusion}

Next-generation sequencing technologies have revolutionized our understanding of molecular mechanisms underlying neurological disorders, including the role of PEs in DEEs. The search for PEs in development and disease is gaining momentum, and a large number of genetic disorders have been identified as a result of disruption of alternative splicing, leading to $\mathrm{PE}$ misregulation. Currently, PE functional studies are limited to mammalian systems, and whether PEs play an evolutionarily conserved role in gene regulation remains unclear. Understanding the role of alternative splicing and PEs in gene regulation across species will help us bridge the gap between preclinical RNA therapies and clinical therapy for NDDs. This is particularly appropriate for the expansion of animal models, beyond just rodents, and the possibilities of zebrafish, frog, and other genetic epilepsy models need to be explored. Targeting PEs with ASOs is efficient in regulating PE inclusion in cellular and animal models, though further development is needed for iPSC-based models for those genes without a good animal epilepsy model. ASO therapies are transforming the treatment of various NDDs, promising to alter the reality of intractable diseases, including DEEs. While these advances provide promising insights into the treatment of DEEs, most therapies remain to be optimized for long-term use.

\section{Conflict of Interest Statement}

G.L.C. holds a collaborative research grant with Stoke Therapeutics. All other authors have no conflicts of interest to declare. 


\section{Funding Sources}

G.L.C. is sponsored by a Dravet Syndrome Foundation Research Grant which sponsors the identification of the RNA-binding proteins that control splicing of the $S C N 1 A$ poison exon.

\section{Author Contributions}

All authors wrote and revised the manuscript.

\section{References}

1 Lareau LF, Inada M, Green RE, Wengrod JC, Brenner SE. Unproductive splicing of SR genes associated with highly conserved and ultraconserved DNA elements. Nature. 2007 Apr 19; 446(7138):926-9.

2 Lareau LF, Brenner SE. Regulation of splicing factors by alternative splicing and NMD is conserved between kingdoms yet evolutionarily flexible. Mol Biol Evol. 2015 Apr;32(4):1072-9.

3 Bejerano G, Pheasant M, Makunin I, Stephen S, Kent WJ, Mattick JS, et al. Ultraconserved elements in the human genome. Science. 2004 May 28;304(5675):1321-5.

4 Königs V, de Oliveira Freitas Machado C, Arnold B, Blümel N, Solovyeva A, Löbbert S, et al. SRSF7 maintains its homeostasis through the expression of split-ORFs and nuclear body assembly. Nat Struct Mol Biol. 2020 Mar;27(3): 260-73.

5 Zhang X, Chen MH, Wu X, Kodani A, Fan J, Doan R, et al. Cell-type-specific alternative splicing governs cell fate in the developing cerebral cortex. Cell. 2016 Aug 25;166(5):1147-e15.

6 García-Rodríguez R, Hiller M, Jiménez-Gracia L, van der Pal Z, Balog J, Adamzek K, et al. Premature termination codons in theDMDgene cause reduced local mRNA synthesis. Proc Natl Acad Sci U S A. 2020 Jul 14;117(28):16456-64.

7 Palombo R, Verdile V, Paronetto MP. Poisonexon inclusion in DHX9 reduces its expression and sensitizes ewing sarcoma cells to chemotherapeutic treatment. Cells. 2020 Jan 31;9(2): 328.

8 Thomas JD, Polaski JT, Feng Q, De Neef EJ, Hoppe ER, McSharry MV, et al. RNA isoform screens uncover the essentiality and tumor-suppressor activity of ultraconserved poison exons. Nat Genet. 2020 Jan;52(1):84-94.

9 Xie Z, Tang L, Xie Z, Sun C, Shuai H, Zhou C, et al. Splicing characteristics of dystrophin pseudoexons and identification of a novel pathogenic intronic variant in the DMD gene. Genes. 2020 Oct 10;11(10):1180.

10 Plummer NW, McBurney MW, Meisler MH. Alternative splicing of the sodium channel SCN8A predicts a truncated two-domain protein in fetal brain and non-neuronal cells. J Biol Chem. 1997 Sept 19;272(38):24008-15.

11 O’Brien JE, Drews VL, Jones JM, Dugas JC, Barres BA, Meisler MH. Rbfox proteins regulate alternative splicing of neuronal sodium channel
SCN8A. Mol Cell Neurosci. 2012 Feb;49(2): 120-6.

12 Veeramah KR, O’Brien JE, Meisler MH, Cheng X, Dib-Hajj SD, Waxman SG, et al. De novo pathogenic SCN8A mutation identified by whole-genome sequencing of a family quartet affected by infantile epileptic encephalopathy and SUDEP. Am J Hum Genet. 2012 Mar 9; 90(3):502-10.

13 Meisler MH, Helman G, Hammer MF, Fureman BE, Gaillard WD, Goldin AL, et al. SCN8A encephalopathy: research progress and prospects. Epilepsia. 2016 Jul;57(7):1027-35.

14 Carvill GL, Engel KL, Ramamurthy A, Cochran JN, Roovers J, Stamberger H, et al. Aberrant inclusion of a poison exon causes Dravet syndrome and related SCN1A-associated genetic epilepsies. Am J Hum Genet. 2018 Dec 6;103(6): 1022-9.

15 Steward CA, Roovers J, Suner MM, Gonzalez JM, Uszczynska-Ratajczak B, Pervouchine D, et al. Re-annotation of 191 developmental and epileptic encephalopathy-associated genes unmasks de novo variants in SCN1A. NPJ Genom Med. 2019;4:31.

16 Heyne HO, Singh T, Stamberger H, Abou Jamra R, Caglayan H, Craiu D, et al. De novo variants in neurodevelopmental disorders with epilepsy. Nat Genet. 2018 Jul;50(7):1048-53.

17 Heyne HO, Artomov M, Battke F, Bianchini C, Smith DR, Liebmann N, et al. Targeted gene sequencing in 6,994 individuals with neurodevelopmental disorder with epilepsy. Genet Med. 2019 Nov;21(11):2496-503.

18 Truty R, Patil N, Sankar R, Sullivan J, Millichap J, Carvill G, et al. Possible precision medicine implications from genetic testing using combined detection of sequence and intragenic copy number variants in a large cohort with childhood epilepsy. Epilepsia Open. 2019 Sept;4(3):397-408.

19 Lim KH, Han Z, Jeon HY, Kach J, Jing E, WeynVanhentenryck S, et al. Antisense oligonucleotide modulation of non-productive alternative splicing upregulates gene expression. Nat Commun. 2020 Jul 9;11(1):3501.

20 Rinaldi C, Wood MJA. Antisense oligonucleotides: the next frontier for treatment of neurological disorders. Nat Rev Neurol. 2018;14(1): 9-21.

21 Bennett CF, Krainer AR, Cleveland DW. Antisense oligonucleotide therapies for neurode- generative diseases. Annu Rev Neurosci. 2019 Jul 8;42:385-406.

22 Anwar A, Saleem S, Patel UK, Arumaithurai K, Malik P. Dravet syndrome: an overview. Cureus. 2019 Jun 26;11(6):e5006.

23 Hawkins NA, Calhoun JD, Huffman AM, Kearney JA. Gene expression profiling in a mouse model of Dravet syndrome. Exp Neurol. 2019 Jan;311:247-56.

24 Han Z, Chen C, Christiansen A, Ji S, Lin Q, Anumonwo C, et al. Antisense oligonucleotides increase $\mathrm{Scn} 1 \mathrm{a}$ expression and reduce seizures and SUDEP incidence in a mouse model of Dravet syndrome. Sci Transl Med. 2020 Aug 26; 12(558): 12 .

25 Chiriboga CA, Swoboda KJ, Darras BT, Iannaccone ST, Montes J, De Vivo DC, et al. Results from a phase 1 study of nusinersen (ISIS$\mathrm{SMN}[\mathrm{Rx}]$ ) in children with spinal muscular atrophy. Neurology. 2016 Mar 8;86(10):890-7.

26 Lenk GM, Jafar-Nejad P, Hill SF, Huffman LD, Smolen CE, Wagnon JL, et al. Scn8a antisense oligonucleotide is protective in mouse models of SCN8A encephalopathy and Dravet syndrome. Ann Neurol. 2020 Mar;87(3):339-46.

27 Sullivan BJ, Kipnis PA, Ammanuel SG, Kadam $\mathrm{SD}$. GABAergic signaling promotes early-life seizures in epileptic SYNGAP1+/- mice. bioRxiv. 2020;11:381749.202014.

28 Kim YJ, Khoshkhoo S, Frankowski JC, Zhu B, Abbasi S, Lee S, et al. Chd2 is necessary for neural circuit development and long-term memory. Neuron. 2018 Dec 5;100(5):1180-e6.

29 Simkin D, Kiskinis E. Modeling pediatric epilepsy through iPSC-based technologies. Epilepsy Curr. 2018 Jul-Aug;18(4):240-5.

30 Barral S, Kurian MA. Utility of induced pluripotent stem cells for the study and treatment of genetic diseases: focus on childhood neurological disorders. Front Mol Neurosci. 2016;9:78.

31 Lancaster MA, Renner M, Martin CA, Wenzel D, Bicknell LS, Hurles ME, et al. Cerebral organoids model human brain development and microcephaly. Nature. 2013 Sept 19;501(7467): 373-9.

32 Tidball AM, Lopez-Santiago LF, Yuan Y, Glenn TW, Margolis JL, Clayton Walker J, et al. Variant-specific changes in persistent or resurgent sodium current in SCN8A-related epilepsy patient-derived neurons. Brain. 2020 Oct 1; 143(10):3025-40. 